\title{
Two Cases of Jaw Reconstruction Using Vascularized Fibular Bone Graft and Implant
}

\author{
Shin Takagi'), Nobuyoshi Mizukawa²), Shoji Fukunaga ${ }^{1)}$, Nobuhisa Ishida'), Toshio Sugawara' ${ }^{1)}$ \\ ${ }^{1)}$ Department of Oral and Maxillofacial Reconstructive Surgery, Graduate School of Medicine, Dentistry and Pharmaceutical Sciences, \\ Okayama University Graduate Schools \\ ${ }^{2)}$ Clinic of Oral and Maxillofacial Reconstructive Surgery, Okayama University Hospital
}

\section{Introduction}

Maxilla reconstruction is conventionally performed using metal plate or free bone graft ${ }^{1)}$. However, conventional methods do not allow occlusal reconstruction to improve the patients' QOL. Recently, vascularized bone grafts have been used in reconstruction of the jaw bone ${ }^{2,3)}$. After the jaw bone is reconstructed, implants are placed aiming to reconstruct occlusion. We report two cases using this reconstruction method.

\section{Case Report}

Case 1: A 41 year-old presented on December 6, 1996. Malignant tumor of maxilla was diagnosed clinically, and squamous cell carcinoma was diagnosed histopathologically. On December 26, 1996, right cervical lymph node dissection and right maxillary hemisection were conducted under general anesthesia. Thereafter, a maxillary prosthesis was worn, but due to blockage of the communication between the buccal cavity and nasal cavity as well as the maxillary sinus, transplantation of a vascularized fibular bone graft was conducted at the Department of Plastic Surgery of the medical school on September 14, 2001. The patient desired to have fixed dental prosthesis. Therefore, on May 1, 2003, five SteriOss implants $(3.8 \mathrm{~mm} \times 10 \mathrm{~mm})$ were implanted into the fibular graft and two $(3.8 \mathrm{~mm} \times 12 \mathrm{~mm})$ implanted into the alveolar bone of the left anterior teeth region under general anesthesia. On September 25, four months after the first operation, the second operation was performed. Thereafter, the upper structure of the dental prosthesis was fabricated at the Dental Prosthesis Department of our hospital, and was attached on March 10, 2005 (Figure 1).

Case 2: A 27 year-old man had a tumor with a histopathological diagnosis of ameloblastoma. On November 5, 1998, segmental resection of the left mandible was performed under general anesthesia, followed by plate reconstruction. Thereafter, a second reconstruction was performed using a free iliac bone graft, which was removed on October 5, 2000 due to infection. On July 24, 2002, vascularized fibular bone grafting was conducted at the Department of Plastic Surgery. On June 22, 2003, three SteriOss implants (two at $3.8 \mathrm{~mm} \times 16 \mathrm{~mm}$, and one at $3.8 \mathrm{~mm} \times 14 \mathrm{~mm}$ ) were implanted into the fibular graft, and a bone graft harvested from the mentum was also transplanted. On November 27, five months after the first operation, the second operation (one abutment $3.8 \mathrm{~mm}$ in diameter and 2 abutments $3.25 \mathrm{~mm}$ in diameter) was performed, and the upper structure of the bridge prosthesis was attached (Figure 2).

\section{Discussion}

When a vascular bone graft is used, since blood circulation is restored immediately after grafting, the remodeling process of bone formation can be omitted, and the graft survives without resorption ${ }^{2,3)}$. In addition, the graft has enough thickness and width for implants as an occlusal reconstruction method. The efficacy of vascular bone graft has been proven ${ }^{1}$, and clinical application has also been reported ${ }^{4,5,6}$. The vascular bone graft may be harvested from various sources including the iliac bone, scapula and fibula, each has different characteristics. The fibula has enough length and contains large amount of compact bone. Fibular bone graft is useful for osseointegration in fixtures, but has the demerit of not providing enough height.

By combining jaw reconstruction with implant surgery, occlusal function is improved with additional cosmetic benefit. This new approach is a good news for the patients. On the other hand, there are also several issues such as inadequate soft tissue around the implant, mobility, long treatment time, and high cost. Topics for future investigations include defining the optimal insertion site of the bone graft, timing of implant placement, and method of occlusal function test.

\section{References}

1. Horikoshi K, Kusama M, Kishi T, Miyazawa S, Ono T, Fujibayashi K, Nakura H, Enomoto S and Harii K.: Nine cases of secondary mandibular reconstruction following surgery for malignant oral tumors. Ni Ko Ge Shi, 35, 2314-2320, 1989.

2. Harii K, Mitsujima I, Sato T, Tsuchiya T and IshiyamaY.: Latest advances of vascular free bone grafting. Sei Ge Saigai Geka,25, 877-888, 1982.

3. Takado T, Harii K, et al: Research on vascular bone graftingFirst report: Comparison with free bone grafting. Ni Kei Kai Chi, 5, 487-504, 1985.

4. Sasaguri M, Higuchi S, Ohseki S, Haita R, Tashiro H and Uchida Y.: Two cases of mandibular reconstruction using vascular free fibular bone graft. Ni Ko Ge Shi, 37, 1732-1738, 1991.

5. Ogiso A, Minemura S, Tamura M, Ogiba A, Kurita H, Kurashina K, Kotani A and Iwasawa M.: Two cases of reconstruction of wide partial mandibular defect using vascular bone grafting. Ni Ko Ge Shi, 38, 1424-1430, 1992.

6. Worthington P and Branemark PI.: Mandibular Reconstruction. 1st ed. Thieme Medical Publishers Inc. New York, 117-131, 1997. 